\title{
LYPD3 Gene
}

National Cancer Institute

\section{Source}

National Cancer Institute. LYPD3 Gene. NCI Thesaurus. Code C132240.

This gene is involved in cell migration. 